\title{
Will use of the Six-Item Cognitive Impairment Test help to close the dementia diagnosis gap?
}

\section{Anne-Marie Cagliarini', Hannah L Price', Sam T Livemore' \& Andrew J Larner*1}

Aim: To measure the frequency of use of the Six-Item Cognitive Impairment Test (6CIT), a local Dementia Commissioning for Quality and Innovation target, prior to patient referral to a dedicated cognitive disorders clinic. Methods: Consecutive new outpatient referral letters were examined over a 6-month period (July-December 2012) for 6CIT use. The final clinical diagnosis was compared with 6CIT score. Frequency of 6CIT use was compared with prior similar surveys in this clinic. Results: Approximately only $5 \%$ of new patients had been administered the 6CIT prior to referral, all from primary care. The 6CIT score correlated with final clinical diagnosis in all cases. The frequency of 6CIT use has increased compared with prior surveys in 2004-2006 and 2009-2010 $(\sim 1 \%)$. Conclusion: If 6 CIT is to facilitate the aims of the Dementia Commissioning for Quality and Innovation in closing the dementia diagnosis gap, more widespread use will be required, with implications for training in its use.

The dementia diagnosis gap refers to the discrepancy between the number of individuals with dementia in the population as predicted on the basis of epidemiological studies and the number actually diagnosed based on returns from primary care practitioners submitted as part of the requirements of the Quality and Outcomes Framework in general practice. Comparisons suggest that perhaps only $40 \%$ of individuals with dementia in the UK have a diagnosis, although this does vary from region to region $[1,2]$. Clearly, those without a proper diagnosis may not receive appropriate interventions and support, including treatment with drugs that are appropriate for dementia syndromes.

The Dementia Commissioning for Quality and Innovation (CQUIN) document, published under the auspices of the UK Government in April 2012, sought to implement a proactive approach to identify people with dementia, in part to address the dementia diagnosis gap [3]. This is to be achieved by asking all individuals aged 75 years or over a screening question ("have you been more forgetful in the past 12 months to the extent that it has significantly affected your life?"), which if answered in the affirmative is, according to the Dementia CQUIN flowchart, to trigger a 'dementia risk assessment' [3]. Compliance with the Dementia CQUIN is incentivized with cash payments by level of performance. Initially targeted at patients presenting in secondary care, the principles of the Dementia CQUIN may also be imposed in primary care [4].
The details of the Dementia CQUIN 'dementia risk assessment' are unspecified, but it would seem likely that administration of some form of cognitive screening instrument would form an integral part of any such assessment. A large number of cognitive screening instruments have been described in the medical literature [5], each with its pros and cons, proponents and detractors. Whereas the Mini-Mental State Examination is well known and longstanding [6], it is probably too unwieldy for routine use, with difficulties in the interpretation of test scores and the establishment of cutoffs, and too time consuming for use particularly in primary care, not to mention the enforcement of copyright issues that now surround its use. Briefer instruments specifically designed for use in primary care include the SixItem Cognitive Impairment Test (6CIT): it takes approximately $2 \mathrm{~min}$ to perform, covers several cognitive domains (Box 1), is easy to score (0-28; a higher score represents a worse performance) and scores are classified to aid test interpretation, as 'normal cognition' (0-4), 'questionable impairment' (5-9) or 'suggesting impairment consistent with dementia and requiring further evaluation' (10 or more) [7,8].

To examine whether the 6CIT might be helpful in improving dementia diagnosis, we undertook a prospective study to measure the frequency of its use based on referral letters to a Cognitive Function Clinic (CFC) based in a regional neuroscience center in Liverpool (UK). The 6CIT has been accepted as a CQUIN target

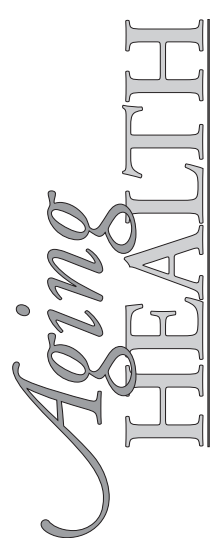

${ }^{1}$ Cognitive Function Clinic, Walton Centre for Neurology \& Neurosurgery, Lower Lane, Fazakerley, Liverpool, L9 7LJ, UK

*Author for correspondence: a.larner@thewaltoncentre.nhs.uk

\section{Keywords}

-6CIT • dementia • Dementia Commissioning for Quality and Innovation • diagnosis

\section{Future :\%: Medicine part o}


Box 1. Item content of the Six-Item Cognitive Impairment Test.

- Orientation (patient asked to state year, month and exact time of day): 10

- Calculation (count backwards from 20 to 1; recite months of the year backwards): 8

- Memory recall (five-item name and address): 10

- Total score: 28

over the last 12 months by two National Health Service Trusts (Liverpool Community Health, Liverpool, UK, and Bridgewater Community Healthcare, Wigan, UK) within the CFC catchment area. Results were compared with previous studies undertaken in CFC looking at usage of cognitive screening instruments prior to referral to $\mathrm{CFC}[9,10]$.

\section{Methods}

In this prospective study, referral letters of consecutive patients seen in CFC over a 6-month period (July-December 2012; following the publication of the Dementia CQUIN) were examined for any mention of use of the 6CIT prior to referral, a method used in previous studies $[9,10]$. If present, the 6CIT score was noted and then compared with the final patient diagnosis (based on judgment of an experienced clinician), but the 6CIT score was not used in the diagnostic judgment in order to avoid review bias. The frequency of 6CIT use was compared with that measured in previous studies of outpatient cohorts from this clinic $[9,10]$.

\section{Results}

Out of 132 new outpatient referrals seen over the 6 -month period $($ male:female $=74: 58,56 \%$ male; age range: $20-88$ years; median: 58 years), 99 were from primary care $(75 \%)$. The referrals from secondary care $(33 ; 25 \%)$ were approximately equally distributed between those from psychiatrists (13), neurologists (9) and other physicians (11). Overall, 41 patients were judged to be demented (using DSM-IV-TR criteria) and a further 23 had mild cognitive impairment (based on modified Petersen criteria), giving a prevalence of dementia of $31 \%$ and of cognitive impairment of $48 \%$, figures similar to those from previous $\mathrm{CFC}$ cohorts. Of the 41 patients with dementia, 27 were referred from primary care $(27 \%$ of primary care referrals) and 14 were from secondary care $(42 \%$ of secondary care referrals). The null hypothesis that the proportion of patients with dementia did not differ significantly by referral source was not rejected, although a trend was observed $\left(\chi^{2}=3.05 ; \mathrm{df}=1\right.$; $0.1>\mathrm{p}>0.05)$.

Use of the 6CIT was mentioned in seven referral letters (5.30\%; 95\% CI: 1.48-9.13). All of these referrals were from primary care. In six out of seven cases, the actual 6CIT score was given (range 0/28-28/28), one letter merely stated the 6CIT was 'normal'. Of these seven referrals, 6CIT score was normal in two and 'consistent with dementia' in five referrals. The final clinical diagnosis in these patients was consistent with 6CIT diagnostic category in six out of seven patients, the one exception being a patient with the a 6CIT score 'consistent with dementia' whose final clinical diagnosis was mild cognitive impairment.

Previous surveys have found very infrequent mention of 6CIT use in referrals to CFC from primary care (TaвLE 1) [9,10]. The current study, therefore, suggested an increase in 6CIT use following publication of the Dementia CQUIN and its acceptance in local National Health Service Trusts. The null hypothesis that the proportion of primary care referrals mentioning 6CIT use did not differ significantly in the different patient cohorts was rejected $\left(\chi^{2}=7.89 ; \mathrm{df}=2\right.$; $\mathrm{p}<0.02)$.

\section{Discussion}

The dementia diagnosis gap remains a significant challenge to healthcare providers aiming to ensure appropriate targeting of high-quality healthcare interventions to individuals with dementia. Previous studies of UK government directives on dementia and its treatment (National Dementia Strategy, NICE 2011 guidance on dementia drug use) have not produced any detectable increase in diagnosis rate, and hence of gap closure, as judged by experience in CFC $[10,11]$. The Dementia CQUIN represents another attempt to close this diagnosis gap, but with its lack of operationalization, beyond a screening question and a flowchart, it remains largely aspirational, and its efficacy has yet to be measured. Recommendation of a specific cognitive screening instrument in the Dementia CQUIN flowchart, such as the 6CIT, might facilitate case finding.

This study found very few referrals to CFC since publication of the Dementia CQUIN had been assessed with the 6CIT prior to referral, although there was an increase in previous cohorts seen in the same clinic. Concerns over possible 6CIT overuse or misuse, which have been expressed locally, currently seem to be 
without foundation. In the few cases seen, the 6CIT scores correlated with final clinical diagnosis, with no examples of false negatives seen. This is in accordance with previous studies suggesting 6CIT has high test sensitivity [5,12], a desideratum for a cognitive screening instrument.

\section{Limitations}

The information collected was from a single clinic over a limited time period, with obvious risk of selection bias, albeit that CFC has a very broad catchment area. In addition, data collection occurred shortly after the publication of Dementia CQUIN, so implementation was perhaps at an early stage. Nonetheless, changes in numbers of clinic referrals have been detected following other UK Government directives on dementia (NICE/SCIE guidelines, National Dementia Strategy) and the monetary incentivization of Dementia CQUIN at a time of financial squeeze may increase the likelihood of its early implementation [10]. An obvious unknown, not accessible to study methodology, is how many patients have been administered 6CIT and not been referred, presumably on the basis of a reassuring score.

We did not examine use of cognitive screening instruments other than 6CIT in this study, but our previous studies have found that only approximately $20 \%$ of referrals to CFC from primary care mention use of any kind of cognitive screening instrument, mostly Mini-Mental State Examination and the Abbreviated Mental Test Score, suggesting that most referral sources do no testing $[9,10]$.

\section{Conclusion}

There is currently no evidence that Dementia CQUIN is any more successful than previous
Table 1. Use of the Six-Item Cognitive Impairment Test in referrals to a Cognitive Function Clinic from primary care, sequential patient cohorts.

\begin{tabular}{|lllr|}
\hline Time frame & Patients (n) & 6CIT use, $\mathbf{n ~ ( \% ) ~}$ & Ref. \\
\hline October 2004-September 2006 & 123 & $1(0.81)$ & {$[9]$} \\
\hline February 2009-February 2010 & 175 & $2(1.14)$ & {$[10]$} \\
July-December 2012 & 99 & $7(7.07)$ & \\
\hline 6CIT: Six-Item Cognitive Impairment Test. & & & \\
\hline
\end{tabular}

UK government directives on dementia in closing the diagnosis gap, but longer-term studies will be required. The absence of operationalization of Dementia CQUIN beyond the screening question may undermine its aspirations. The 6CIT is a brief cognitive screening instrument that is easy to score and interpret, use of which may facilitate the Dementia CQUIN goals. This study showed that the 6CIT is still very infrequently used prior to patient referral to CFC, although its use may be increasing in primary care as a consequence of local recommendations for its use as a Dementia CQUIN target. The limited data suggest that 6CIT is probably effective in identifying patients with cognitive impairment who require onward referral and assessment in dedicated memory clinics. If the 6CIT is to facilitate the aims of the Dementia CQUIN in closing the dementia diagnosis gap, more widespread use will be required, with implications for training in its use.

\section{Future perspective}

With the aging of the population, dementia prevalence will increase in future years. Without appropriate case-finding strategies, the dementia diagnosis gap may grow. If this gap is to be bridged in the next 5-10 years, more will need to be done

\section{Executive summary}

\section{Background}

- The dementia diagnosis gap - fewer patients diagnosed with dementia than anticipated based on epidemiology - remains significant in the UK.

- The UK government Dementia Commissioning for Quality and Innovation, published in April 2012, aims to identify individuals with cognitive impairment by opportunistic use of a screening question.

- The Six-Item Cognitive Impairment Test $(6 \mathrm{CIT})$ is a brief cognitive screening instrument that may be suitable for use as part of the Dementia Commissioning for Quality and Innovation case-finding strategy.

\section{Method}

- Consecutive referral letters to a Cognitive Function Clinic were examined for evidence of 6CIT use prior to referral.

\section{Results}

- Approximately only $5 \%$ of referral letters mentioned 6CIT use, all from primary care.

- 6CIT scores correlated with final clinical diagnosis, with no false negatives.

\section{Conclusion}

- This study suggests that 6CIT use is currently still very limited, with negative implications for achieving Dementia Commissioning for Quality and Innovation goals and reducing the dementia diagnosis gap. 
to develop public awareness and, critically, to improve use of cognitive screening instruments in the primary care setting to identify patients with cognitive decline who may benefit from referral for treatment and/or intervention options. Increased use of the 6CIT, or a similar brief cognitive screening instrument, may facilitate this, but more training will need to be given to ensure that it can be appropriately applied in primary care. Such tools may complement efforts to increase public awareness of dementia, along with robust education programs to recognize the need for people to start on the diagnosis pathway. The current study, along with previous studies in this setting $[10,11]$, provides no evidence to indicate that centrally issued dementia policy directives have any significant impact in closing the dementia diagnosis gap, perhaps related to their aspirational nature and lack of explicit operationalization. We think it unlikely that future policy directives will meet with any greater success, unless one of the central tenets of the WHO criteria for disease screening is fulfilled, namely availability of a treatment for the condition that is more beneficial when applied at the presymptomatic stage compared with the later symptomatic stage (i.e., disease-modifying treatment).

\section{Financial \& competing interests disclosure}

The authors have no relevant affiliations or financial involvement with any organization or entity with a financial interest in or financial conflict with the subject matter or materials discussed in the manuscript. This includes employment, consultancies, honoraria, stock ownership or options, expert testimony, grants or patents received or pending, or royalties.

No writing assistance was utilized in the production of this manuscript.

\section{References}

Papers of special note have been highlighted as:

- of interest

1. Alzheimer's Society. Mapping the Dementia Gap: Study Produced by Tesco, Alzheimer's Society and Alzheimer's Scotland. Alzheimer's Society, London, UK (2011).

2. Alzheimer's Society. Mapping the Dementia Gap 2012. Progress on Improving Diagnosis of Dementia 2011-2012. Alzheimer's Society, London, UK (2013).

- Surveys showing the extent of the dementia diagnosis gap in various regions of the UK.

3. Department of Health. Using the Commissioning for Quality and Innovation (CQUIN) Payment Framework. Guidance on the New National Goals 2012-2013. Department of Health, London, UK (2012).

4. Brunet MD, McCartney H, Heath I et al. There is no evidence base for proposed dementia screening. BMJ345, e8588 (2012).
5. Larner AJ. Cognitive Screening Instruments: A Practical Approach. Springer, London, UK (2013).

6. Folstein MF, Folstein SE, McHugh PR. 'Mini-Mental State'. A practical method for grading the cognitive state of patients for the clinician. J. Psychiatr. Res. 12, 189-198 (1975).

7. Brooke P, Bullock R. Validation of a 6 item cognitive impairment test with a view to primary care usage. Int. J. Geriatr. Psychiatry 14, 936-940 (1999).

8. Jefferies K, Gale TM. 6-CIT: six-item cognitive impairment test. In: Cognitive Screening Instruments: a Practical Approach. Larner AJ (Ed.). Springer, London, UK, 209-218 (2013).

9. Fisher $\mathrm{CAH}$, Larner AJ. Frequency and diagnostic utility of cognitive test instrument use by GPs prior to memory clinic referral. Fam. Pract. 24, 495-497 (2007).

- Study showing the low level of cognitive screening instrument use in referrals to a Cognitive Function Clinic from primary care, suggesting that most referral sources do no such testing.

10. Menon R, Larner AJ. Use of cognitive screening instruments in primary care: the impact of national dementia directives (NICE/SCIE, National Dementia Strategy). Fam. Pract. 28, 272-276 (2011).

- Study showing the low level of cognitive screening instrument use in referrals to a Cognitive Function Clinic from primary care, suggesting that most referral sources do no such testing.

11. Larner AJ. Impact of the 2011 NICE guidance on dementia drugs in a neurology-led memory clinic. Clin. Med. 12, 496 (2012).

12. Tuijl JP, Scholte EM, de Craen AJM, van der Mast RC. Screening for cognitive impairment in older general hospital patients: comparison of the six-item cognitive impairment test with the Mini-Mental State Examination. Int. J. Geriatr. Psychiatry 27, 755-762 (2012). 